Int. J. Dev. Biol. 58: 663-668 (2014)

doi: $10.1387 / \mathrm{ijdb} .140261 \mathrm{rn}$

\title{
Preformed Wolffian duct regulates Müllerian duct elongation independently of canonical Wnt signaling or Lhx1 expression
}

\author{
MASAHIKO CHIGA ${ }^{1,2}$, TOMOKO OHMORI ${ }^{1}$, TAKASHI OHBA ${ }^{2}$, HIDETAKA KATABUCHI $^{2}$ \\ and RYUICHI NISHINAKAMURA*,1 \\ ${ }^{1}$ Department of Kidney Development, Institute of Molecular Embryology and Genetics, Kumamoto University and \\ ${ }^{2}$ Department of Obstetrics and Gynecology, Faculty of Life Sciences, Kumamoto University, Kumamoto, Japan
}

\begin{abstract}
The Müllerian duct gives rise to female reproductive organs, such as the oviduct and uterus. During gestation, the Wolffian duct, which generates male reproductive organs and the kidney, is formed, and the Müllerian duct then elongates caudally along the preformed Wolffian duct. Anatomical separation of these two ducts in chick embryos demonstrated that the Wolffian duct is required for Müllerian duct formation. Likewise, a few reports supported this notion in mice, including studies on Wnt9b mutant mice and Wolffian duct-specific $L h \times 1$ deletion. However, anatomical ablation of the Wolffian duct has not been established in mice. In this study, we addressed the importance of the interaction between these two reproductive ducts, by generating mice that specifically expressed a diphtheria toxin subunit in the Wolffian duct. While this genetic ablation of the Wolffian duct resulted in kidney hypoplasia/agenesis in both male and female mutant mice, the female mutant mice lacked the uterus, which is derived from the Müllerian duct. At mid-gestation, the Müllerian duct was truncated at the level where the mutant Wolffian duct was prematurely terminated, meaning that Müllerian duct elongation was dependent on the preformed Wolffian duct. However, Wnt9b expression in the Wolffian duct and the resultant canonical Wnt activity, as well as Lhx1 expression, were not affected in the mutant mice. These results suggest that the Wolffian duct regulates Müllerian duct elongation by currently unidentified mechanisms that are independent of canonical Wnt signaling or Lhx1 expression.
\end{abstract}

KEY WORDS: Wolffian duct, Müllerian duct, Wnt9b, Lhx1

It has been shown that a subset of human patients with uterus hypoplasia display kidney hypoplasia (Oppelt et al., 2007; Woolf and Allen, 1953). While the uterus and the kidney might partially employ similar developmental programs, an interaction between the precursor tissues may also be involved in the formation of these two organs. The reproductive organs in males and females are derived from the Wolffian duct and Müllerian duct, respectively (Kobayashi and Behringer, 2003). The Wolffian duct (mesonephric duct) is first detected at embryonic day (E) 8.5 in mice, and elongates caudally until it reaches the cloaca by E10.5. At E10.5, the ureteric bud stems out from the Wolffian duct, and contributes to the formation of the kidney through a mutual interaction with the metanephric mesenchyme (Nishinakamura, 2008). While most parts of the Wolffian duct degenerate during development, the epididymis and vas deferens in males are derived from the residual Wolffian duct. The Müllerian duct (paramesonephric duct) emerges at E11.5 as an invagination of the peritoneal cavity, and elongates along the preformed Wolffian duct. The Müllerian duct elongation is completed when it reaches the urogenital sinus at E13.5. The Müllerian duct eventually forms the oviduct, uterus, and upper third of the vagina in females, while it starts to degenerate in males between E13 and E14 through the effects of Müllerian inhibiting substance, which belongs to the TGF- $\beta$ superfamily.

The elongation of the Müllerian duct depends on the preformed Wolffian duct. In chicks, physical elimination of the Wolffian duct or separation of the two ducts using aluminum foil or other methods results in impaired elongation of the Müllerian duct (Bishop-Calame, 1966; Kobayashi and Behringer, 2003). In mice, genetic deletion of genes encoding transcription factors, such as Pax2, $L h x 1$, and Emx2, results in absence of both the Wolffian duct and Müllerian duct (Miyamoto et al., 1997; Shawlot and Behringer, 1995; Torres et al., 1995). Since these genes are expressed in both ducts, it is difficult to discriminate whether the absence of the Müllerian duct is caused by cell-autonomous requirements of these genes in the

Abbreviations used in this paper: E, embryonic day; md, Müllerian duct; wd, Wolffian duct. *Address correspondence to: Ryuichi Nishinakamura. Department of Kidney Development, Institute of Molecular Embryology and Genetics, Kumamoto University,
2-2-1 Honjo, Kumamoto 860-0811, Japan. Tel: +81-96-373-6615. Fax: +81-96-373-6618. E-mail: ryuichi@ kumamoto-u.ac.jp

ISSN: Online 1696-3547, Print 0214-6282 
Müllerian duct or by non-cell-autonomous effects caused by loss of the Wolffian duct. Indeed, Müllerian duct-specific $L h x 1$ deletion revealed that this gene is required cell-autonomously in the Müllerian duct (Huang et al., 2014). The Müllerian duct tip is considered to contain progenitor-like cells with migratory capacity, which may be responsible for the Müllerian duct elongation. Wolffian duct-specific Lhx 1 deletion impairs not only Wolffian duct formation, but also Müllerian duct elongation (Kobayashi et al., 2005), suggesting that the non-cell-autonomous requirement of the Wolffian duct for Müllerian duct elongation also holds true in mice, although the factors that mediate this Lhx1-dependent process remain unknown.

$W n t 9 b$ is expressed in the Wolffian duct and ureteric bud, and evokes the mesenchymal-to-epithelial transition in neighboring tissues (Carroll et al., 2005). Wnt9b in the ureteric bud induces the metanephric mesenchyme to form kidney tubules, while that in the Wolffian duct induces the surrounding mesenchyme to form the Müllerian duct. Therefore, deletion of Wnt9b affects kidney formation, as well as Müllerian duct elongation. Since the formation of the Wolffian duct is not impaired in Wnt9b mutant mice, Wnt9b serves
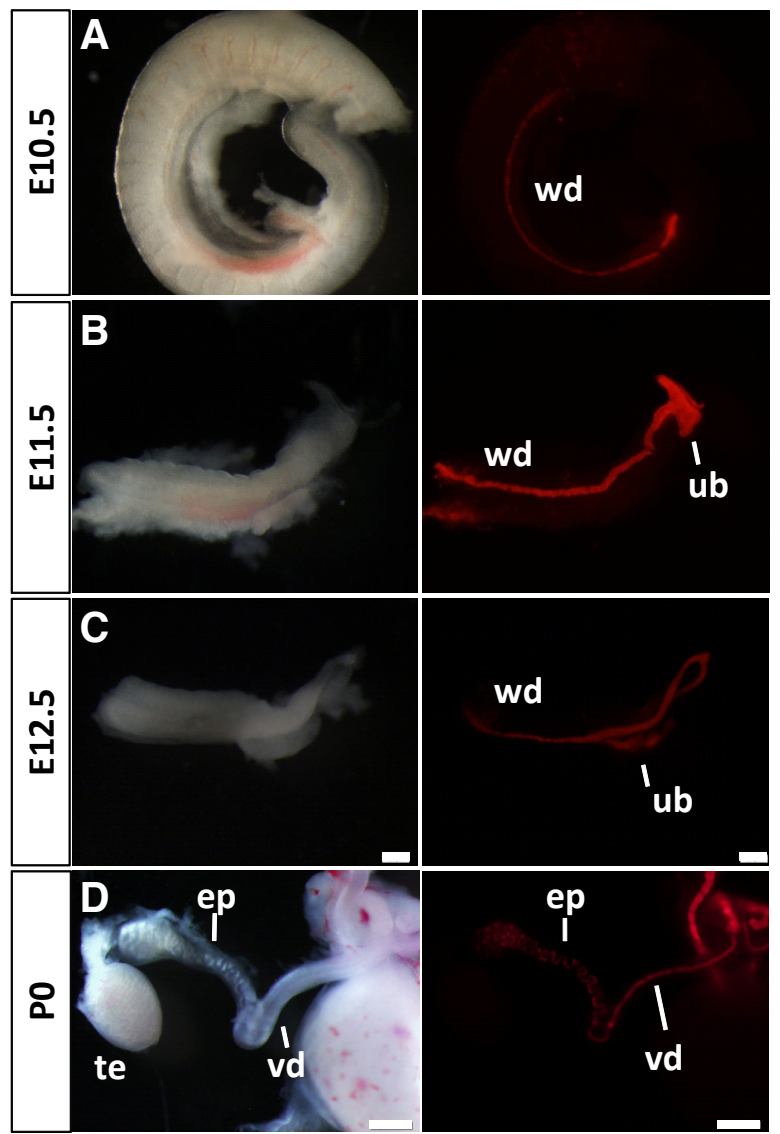

as the only known paracrine molecule to date to explain the noncell-autonomous effects of the Wolffian duct against Müllerian duct elongation. However, the precise mechanisms of Müllerian duct elongation remain largely unknown. Additionally, physical elimination of the Wolffian duct has not been tested in mice. Therefore, we genetically ablated the Wolffian duct by Wolffian duct-specific expression of a diphtheria toxin subunit, and examined the effects on Müllerian duct elongation.

\section{Results}

Hoxb7Cre is specifically expressed in the Wolffian duct

Although Hoxb7Cre mice are widely used for gene deletion in the Wolffian duct and its derivatives (Yu et al., 2002), we first tried

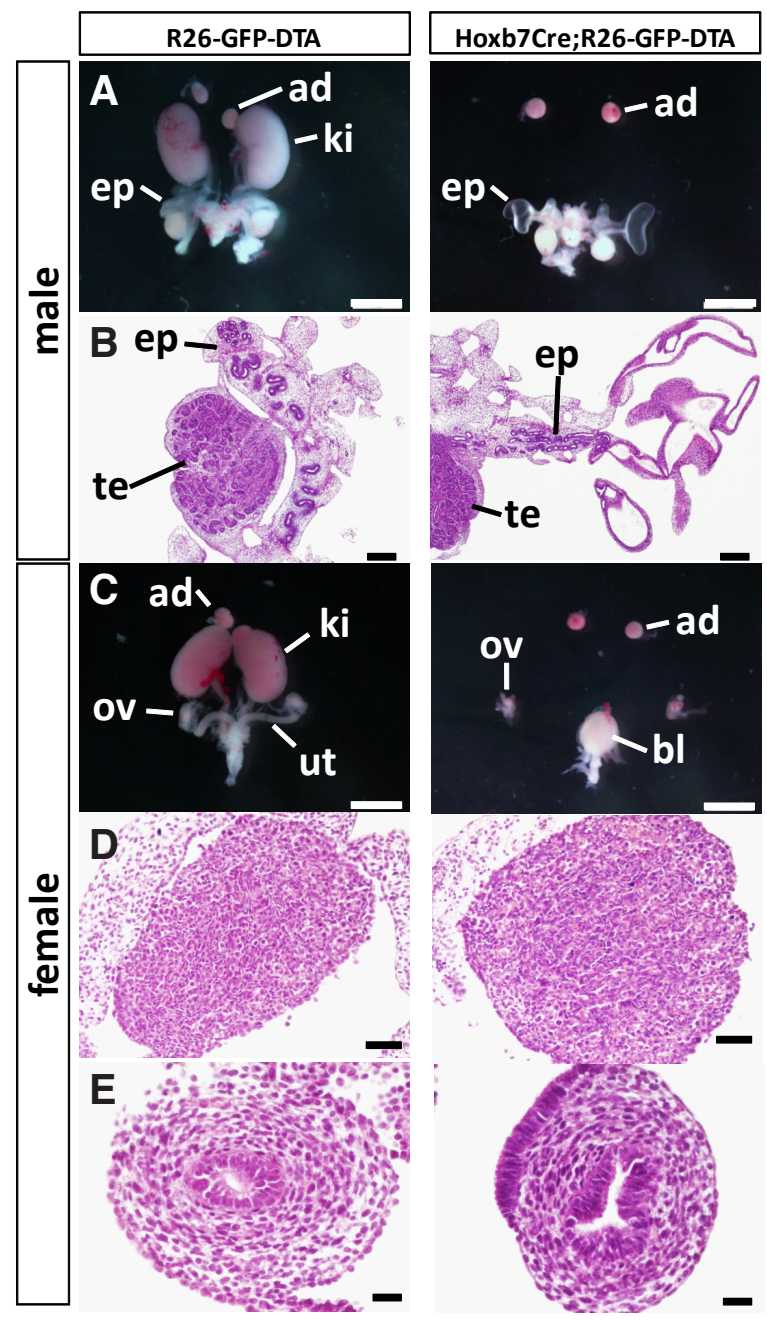

Fig. 1 (left). Hoxb7Cre is specifically expressed in the Wolffian duct. tdTomato expression in Hoxb7Cre; R26-tdTomato mice at E10.5, 11.5, 12.5, and PO. Left columns: bright-field images are partly overlaid with tdTomato signals (right columns). ep, epididymis; te, testis; ub, ureteric bud; vd, vas deferens; wd, Wolffian duct. Scale bars (A-C), 200 um; (D) 500 um.

Fig. 2 (right). Wolffian duct ablation causes organ abnormalities derived from both the Wolffian duct and Müllerian duct. (A) Male urogenital organs in control (R26-GFP-DTA) and mutant (Hoxb7Cre; R26-GFP-DTA) mice at P0. The kidneys (ki) are absent and the epididymis (ep) is dilated. ad: adrenal gland. Scale bar: $2 \mathrm{~mm}$. (B) HE-stained frontal sections of the testes (te) and epididymis (ep). Scale bar: 200 um. (C) Female urogenital organs in control (R26-GFP-DTA) and mutant (Hoxb7Cre; R26-GFP-DTA) mice at PO. The kidneys (ki) and uterus (ut) are absent, while the ovaries (ov) and urinary bladder (bl) are retained. Scale bar: $2 \mu \mathrm{m}$. (D) HE-stained frontal sections of the ovaries. Scale bar: $50 \mu \mathrm{m}$. (E) HE-stained frontal sections of the oviducts. Scale bar: $20 \mu \mathrm{m}$. 
to eliminate the possibility of ectopic activity, especially in the Müllerian duct. We crossed Hoxb7Cre mice with a reporter strain in which the CAG promoter, floxed stop sequences, and tandem dimer Tomato (tdTomato) coding sequence were inserted into the Rosa26locus (Madisen etal., 2010). At E10.5, tdTomato expression was specifically detected in the Wolffian duct (Fig. 1A). tdTomato continued to be expressed in the Wolffian duct, as well as in the kidney-forming ureteric buds, at E11.5 and E12.5 (Fig. 1 B,C), and no ectopic signals were detected. In newborn (P0) males, tdTomato was expressed in the seminiferous tubules, which connect the testis to the excretory route (Fig. 1D). These results reflect the spatially-restricted activity of Cre recombinase in Hoxb7Cre mice, in that Hoxb7Cre is specifically expressed in the Wolffian duct and its derivatives during development.

\section{Wolffian duct ablation causes organ abnormalities derived from both the Wolffian duct and Müllerian duct}

To ablate the Wolffian duct genetically, we crossed Hoxb7Cre mice with R26-GFP-DTA mice, in which floxed green fluorescent protein (GFP) sequences and the diphtheria toxin A subunit (DTA) coding sequence were inserted into the Rosa26 locus (Ivanova et al., 2005). Cre recombinase excises the GFP cassette, and activates the downstream DTA, thereby efficiently eliminating the Wolffian duct. The resultant newborn mice exhibited hypoplasia or agenesis of the kidneys in all males and females examined ( $n=4$ for each), because the kidney is formed through reciprocal interactions between the metanephric mesenchyme and the Wolffian duct-derived ureteric buds (Fig. $2 \mathrm{~A}, \mathrm{C}$ ). The mutant male mice (four of four) also showed significantly dilated epididymis (Fig. 2B), although the cause of this phenotype remains uninvestigated. Additionally, the mutant female mice (four of four) lacked the uterus, cervix, and upper portion of the vagina, all of which are derived from the Müllerian duct (Fig. 2C). In contrast, the ovary and lower portion of the vagina, which are not derived from the Müllerian duct, developed normally (Fig. 2D and data not shown). Thus, genetic ablation of Wolffian duct-derived tissues caused defects in Müllerian duct-derived organs in female mice. The intact oviducts (Fig. 2E) were likely to result from residual formation of the Müllerian duct, as described for Fig. 3.

\section{Müllerian duct elongation depends on the preformed Wolff- ian duct}

We further examined the elongation of the Wolffian and Müllerian ducts during development. The Wolffian duct in control mice had already elongated and reached the caudal region by $\mathrm{E} 12.5$, as shown by Wnt9b expression (Fig. 3 A,B, left columns). However, in Hoxb7Cre; R26-GFP-DTA mice, the Wnt9b-positive Wolffian duct ceased to elongate at the level corresponding to the middle portion of the gonads (Fig. $3 \mathrm{~A}, \mathrm{~B}$, right columns), indicating successful ablation of the Wolffian duct. This finding is consistent with kidney agenesis observed in newborn mutant mice (Fig. 2 A,C), because the ureteric bud that should be derived from the caudal end of the Wolffian duct is likely to be absent. The residual formation of the Wolffian duct may reflect the possibility that not all of the duct epithelial cells expressed Cre recombinase. Indeed, this transgenic mouse strain uses only a 1.3-kb enhancer/promoter of Hoxb7 to drive Cre (Yu et al., 2002). It is also noteworthy that Wnt9b expression was retained in the residual Wolffian duct.

Next, we used an $L h x 1$ probe to visualize the Müllerian duct, and less weakly the Wolffian duct. At E12.5, the Müllerian duct in control mice was in the elongation process, and reached the caudal end by E13.5 (Fig. 3 C,D, left columns). However, elongation of the mutant Müllerian ducts was halted at the same level where the Wolffian ducts were terminated (Fig. 3 C,D, right columns). We did not observe significant differences in this phenotype between male and female embryos ( $n=12$ at $E 12.5$ and $n=6$ at $E 13.5)$, partly because degeneration of the Müllerian duct started between E13 and E14. Thus, Müllerian duct elongation was dependent on the preformed Wolffian duct. Although Lhx1 in the Wolffian and Müllerian ducts is essential for Müllerian duct elongation (Huang et al., 2014; Kobayashi et al., 2005), the unaltered Lhx1 expression observed in both ducts of the mutant mice indicates that loss of $L h x 1$ expression is not the major cause of the Müllerian duct defects.

\section{Lhx1 expression is not affected in Müllerian duct epithelia}

The caudal tip of the elongating Müllerian duct is in physical contact with the Wolffian duct. This tip region contains proliferating progenitors that, at least partly, contribute to duct elongation (Orvis

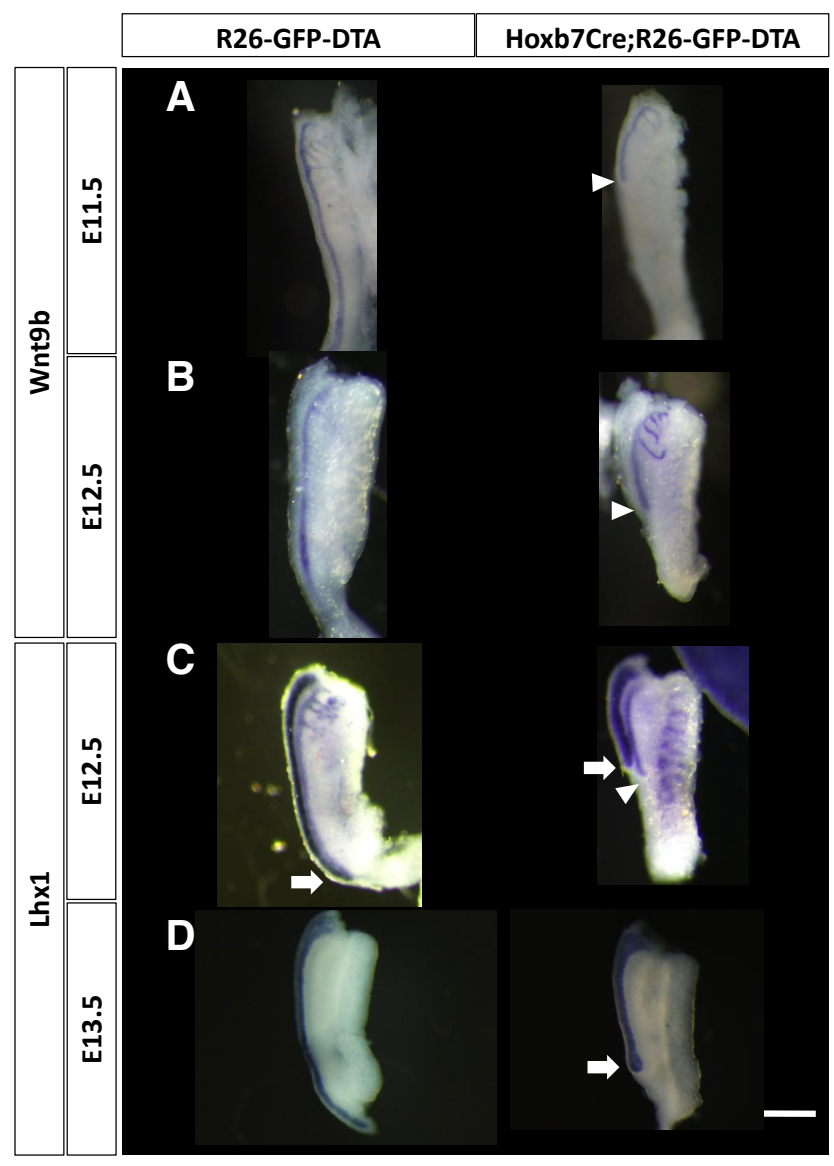

Fig. 3. Müllerian duct elongation depends on the preformed Wolffian duct. $(\mathbf{A}, \mathbf{B})$ Whole-mount in situ hybridization of Wnt9b at E11.5 and E12.5 in control (R26-GFP-DTA) and mutant (Hoxb7Cre; R26-GFP-DTA) embryos. The Wolffian ducts are prematurely terminated (arrowheads) (C,D) Whole-mount in situ hybridization of Lhx1 at E12.5 and E13.5 in control and mutant embryos. The Müllerian ducts elongate up to the point where the Wolffian ducts are terminated (arrowheads). Arrows: Müllerian duct tips. Scale bar, $200 \mu \mathrm{m}$ 
and Behringer, 2007). It is also reported that the tip cells exhibit dynamic morphological changes, forming and retracting multiple cytoplasmic extensions, which suggests a migratory activity of the tip cells (Huang et al., 2014). Thus, we performed histological examinations using sections of the tip region of the elongating Müllerian duct at E12.5. Proliferation of the Müllerian epithelia in the mutant embryos was not significantly impaired, as measured by bromodeoxyuridine (BrdU) incorporation (Fig. 4 A,B). The percentage of BrdU-positive Müllerian duct tips was 27.2 $\pm 12.6 \%$ (455 cells counted) and $16.7 \pm 12.3 \%$ (262 cells counted) in control and mutant embryos, respectively $(n=4$ and $n=3 ; p=0.17)$. Very few apoptotic cells were detected in the Müllerian duct in both control and mutant mice, while some apoptotic cells were detected in the Wolffian duct (Fig. 4C). The percentage of TUNEL-positive cells at the tip of the Müllerian duct was $0.4 \pm 0.7 \%$ (163 cells counted)

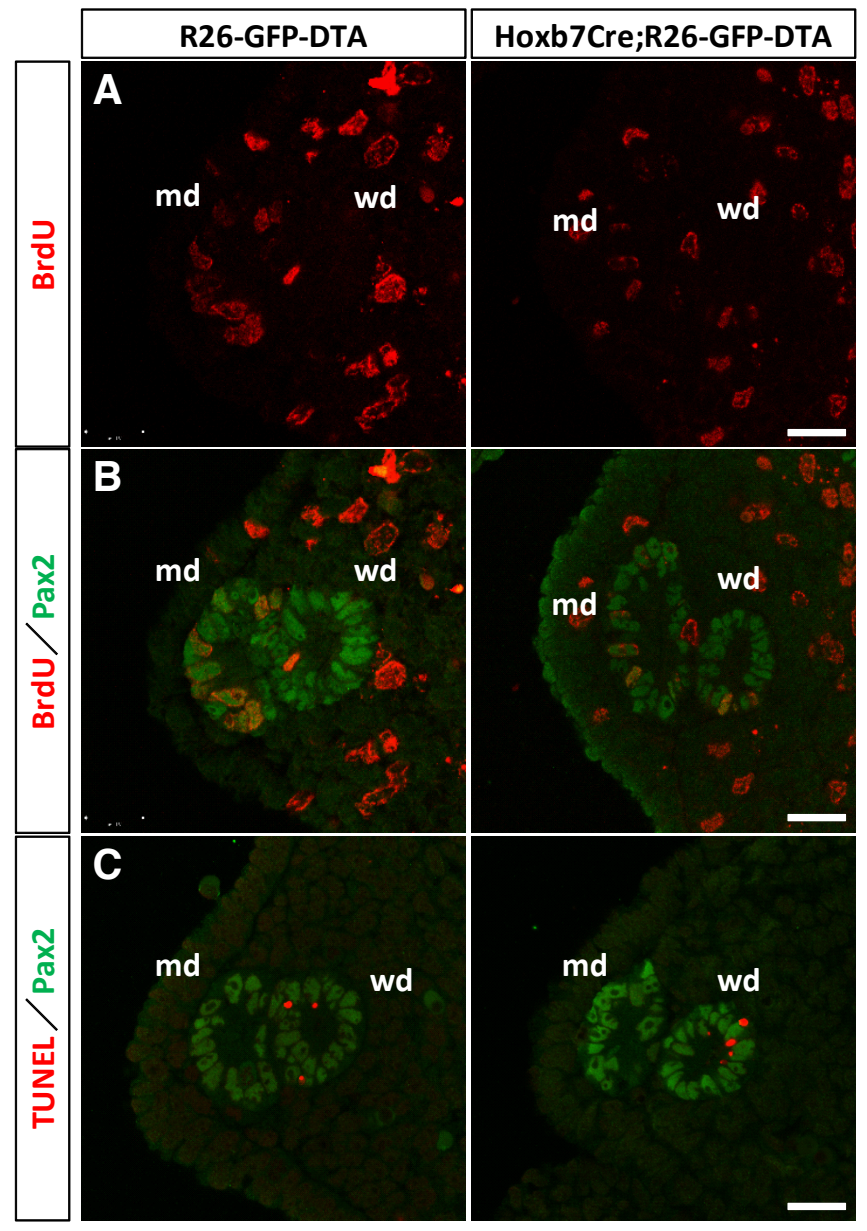

and $2.5 \pm 3.4 \%$ (204 cells counted) in control and mutant mice, respectively ( $n=3$ and $n=4 ; p=0.27$ ). In these experiments, Pax2 was co-stained to identify both the Wolffian and Müllerian ducts. Pax2 is important for Müllerian duct development (Torres et al., 1995), but its expression was not impaired in mutant embryos (Fig. 4 B,C). Therefore, our data suggest that Müllerian duct truncation may be caused by other mechanisms, possibly including a migration defect of the tip cells. Indeed, we noticed that the tip of

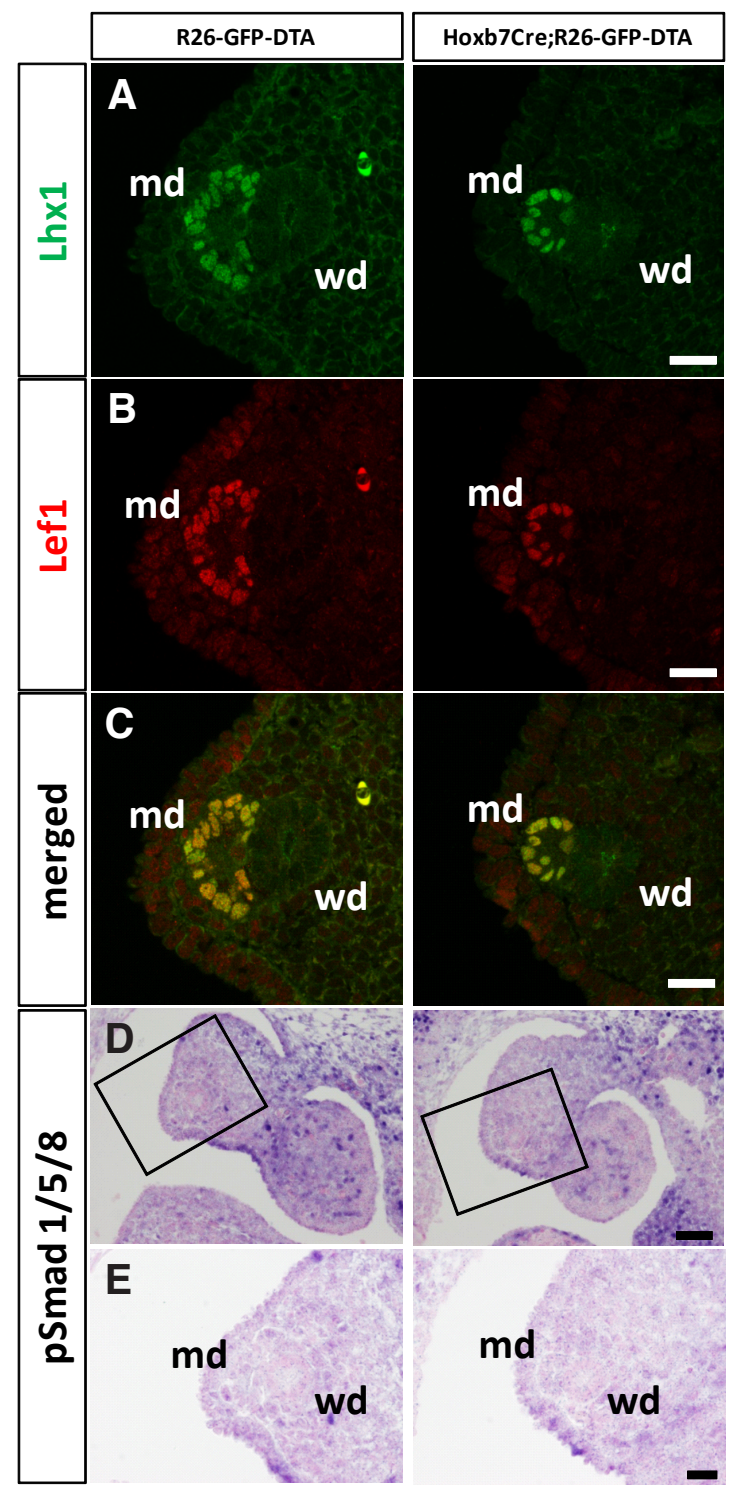

Fig. 4 (left). Proliferation or survival is not affected in Müllerian duct epithelia. (A,B) BrdU (red) and Pax2 (green) staining in control (R26-GFP-DTA) and mutant (Hoxb7Cre; R26-GFP-DTA) embryos at E12.5. Significant proliferation defects are not observed in the mutant embryos. (C) TUNEL (red) and Pax2 (green) staining in control (R26-GFP-DTA) and mutant (Hoxb7Cre; R26-GFP-DTA) embryos at E12.5. Apoptotic cells are not increased in the mutant Müllerian ducts. md, Müllerian duct; wd, Wolffian duct. Scale bar, 20 um.

Fig. 5 (right). Lhx1 expression and canonical Wnt signaling are not affected in the mutant Müllerian tips. (A) Lhx1 staining (green) in control (R26-GFP-DTA) and mutant (Hoxb7Cre; R26-GFP-DTA) embryos at E12.5. Lhx1 and Lef1 are co-stained, but each color is presented separately. Although Lhx1 is expressed in both Wolffian and Müllerian ducts, the antibody that we used only stained the Müllerian duct, which may reflect the different expression levels of the protein. (B) Lef1 staining (red) at E12.5. The same sections in (A) were subjected to double-staining. Note that Lef1 staining is detected only in the Müllerian duct epithelia, but not in the Wolffian ducts. (C) Merged images of Lhx1 and Lef1 staining. (D) pSmad1/5/8 staining (blue signal) at E12.5. Nuclei were counterstained with Nuclear Fast Red. (E) Higher magnification of (D). Signals are undetectable in both the control and mutant Müllerian ducts. md, Müllerian duct; wd, Wolffian duct. Scale bars, (A-C, E) $20 \mu \mathrm{m}$, (D) $50 \mu \mathrm{m}$. 
the mutant Müllerian duct was round and piled up, compared with the tapered form in control mice, as shown by in situ hybridization of Lhx1 (Fig. 3D). This observation is reminiscent of Müllerian duct-specific Lhx1 mutant mice, which show impaired migratory activity in the tip regions (Huang et al., 2014). However, our mutant mice retained $L h x 1$ expression, which was confirmed by section immunostaining (Fig. $5 \mathrm{~A}, \mathrm{C}$ ), as well as by in situ hybridization as shown in Fig. 3D. The unaltered $L h x 1$ expression observed in both ducts of the mutant mice indicates that loss of $L h x 1$ expression is not the major cause of the Müllerian duct defects.

\section{Canonical Wnt signaling is not affected in the mutant Mül- lerian duct tips}

Wnt9b is expressed in the Wolffian duct and its deletion results in absence of the Müllerian duct (Carroll et al., 2005). This process of Müllerian duct formation, as well as tubule formation in the kidney, is considered to be mediated by the mesenchymal-to-epithelial transition evoked by the $\beta$-catenin/Lef1-dependent canonical Wnt pathway. Indeed, Lef1 staining was detected in epithelia of the control Müllerian duct tip regions, but not those of the Wolffian duct (Fig. 5B, C, left panel), suggesting that only the Müllerian duct epithelia respond to Wnt stimuli at this elongation stage. Despite the partial ablation of the Wolffian duct, the remaining duct expressed Wnt9b (Fig. 3A, B), and Lef1 expression in the mutant Müllerian duct tip was not reduced (Fig. 5B, right panel, $n=4$ ). Therefore, the canonical Wnt signaling evoked by Wnt9b and other possible ligands is unlikely to explain the elongation defects of the Müllerian duct in the mutant mice.

We also analyzed phosphorylated Smad1/5/8 (pSmad1/5/8), a downstream effector of Bmp signaling. However, we did not detect any signals in control mice, and no increased staining was observed in mutant mice (Fig. 5D,E). Therefore, currently unidentified mechanisms could exist to explain the Müllerian elongation defects upon Wolffian duct truncation.

\section{Discussion}

We genetically ablated the Wolffian duct and demonstrated that the preformed Wolffian duct is required for proper Müllerian duct elongation. Although $L h x 1$ is required for this process in both cell-autonomous and non-cell-autonomous manners, Lhx1 expression in the mutant mice was not impaired. We also ruled out major extracellular signals, such as Wnt and Bmp, meaning that the Wolffian duct may regulate Müllerian duct elongation by currently unidentified mechanisms.

It is generally considered that the duct tip tends to possess mesenchyme-like characteristics with high migration ability, while the stalk region forms a tight layer of epithelia by a similar mechanism to the mesenchymal-to-epithelial transition. Canonical Wnt activity often enhances the static nature of the epithelia, while the non-canonical pathway promotes cell migration. According to the GUDMAP database (Little et al., 2007), many Wnt ligands, as well as the frizzled ( $f z$ ) receptors, are expressed in both the Müllerian and Wolffian ducts during mid-gestation. These ligands include Wnt5a, Wnt7b, and Wnt9b in the Wolffian duct, and Wnt4, Wnt5a, Wnt7a, and Wnt7b in the Müllerian duct (http://www.gudmap.org/). Our data showing exclusive Lef1 staining in the Müllerian duct epithelia in both the control and mutant embryos suggest that only the Müllerian duct responds to these ligands and that the canonical Wnt pathway is unlikely to explain the phenotype of the mutant mice. However, it is still possible that the non-canonical Wnt pathway plays a role in tip migration. At present, active forms of small GTPases (Rho, Rac, or Cdc42) involved in the non-canonical pathway and migration can only be measured by biochemical approaches that require large numbers of cells. Considering the very small size of the Müllerian duct tip, reagents or techniques that can detect the non-canonical activity should be developed to unequivocally determine whether the non-canonical pathway is responsible for the mutant phenotype.

Shh is expressed in the Wolffian duct, and could be one of the candidate molecules that we have not tested. However, Wolffian duct-specific deletion of Shh has no effects on Müllerian duct elongation (Murashima et al., 2014). Other possibilities include cell-cell contacts, instead of soluble factors, considering the close proximity between the Müllerian and Wolffian ducts. Indeed, a role of fibronectin in Wolffian duct formation was reported in chickembryos (Yoshino et al., 2014). According to the GUDMAP database, integrin $\beta 7$ is expressed in the Müllerian duct, while nephronectin is expressed in the Wolffian duct. Given the importance of nephronectin in the interactions between the metanephric mesenchyme and the Wolffian duct-derived ureteric bud (Linton et al., 2007), it may be worth examining the roles of this type of adhesion molecule in Müllerian duct elongation. However, the currently available datasets have used entire ducts that are anatomically dissected. Identification of markers for the Müllerian duct tip and subsequent generation of GFP-knockin mice, followed by microarray analyses, will help to elucidate the detailed mechanisms of Müllerian duct elongation. Although the precise mechanisms remain to be solved, our mouse model will serve as a useful tool to analyze the interaction between these two reproductive systems.

It was reported that a subset of human patients with uterus hypoplasia display kidney hypoplasia (Oppelt et al., 2007; Woolf and Allen, 1953). At least some of these cases may be derived from primary defects in the Wolffian duct, which affects the Müllerian duct in a non-cell-autonomous manner, similar to the mouse model described in this paper. Thus, any genes that cause developmental arrest of the Wolffian duct may lead to malformation of the uterus and kidney. Alternatively, several genes, including $L h x 1$, are shared for the development of both the Wolffian and Müllerian ducts, and therefore mutations in one particular gene could impair the formation of both organs. Emerging exome sequencing techniques will help to identify the responsible genes in human diseases, which will further accelerate our understanding of the formation of these two reproductive duct systems.

\section{Materials and Methods}

\section{Generation of mutant mice}

Hoxb7Cre, R26-GFP-DTA, and R26-tdTomato mice were obtained from the Jackson Laboratory (Ivanova et al., 2005; Madisen et al., 2010; Yu et al., 2002). The primers used for genotyping were as follows: Cre1 (5'-AGGTTCGTTCACTCATGGA-3') and Cre2 (5'-TCGACCAGTTTAGTTACCC-3') for the Creallele (250-bp product); DTA-mt (5'-GCGAAGAGTTTGTCCTCAACC-3'), DTA-F (5'-AAAGTCGCTCTGAGTTGTTAT-3'), and DTA-R (5'-GGAGCGGGAGAAATGGATATG-3') for the R26R-GFP-DTA allele (wild-type: 600-bp product; mutant: 340-bp product); Tomato-1 (5'-AAGGGAGCTGCAGTGAGTA-3'), Tomato-2 (5'-CCGAAAATCTGTGGGAAGTC-3'), Tomato-3 (5'-GGCATTAAAGCAGCGTATCC-3'), and Tomato-4 (5'-CTGTTCCTGTACGGCATGG-3') for the R26-tdTomato 
allele (wild-type: 297-bp product; mutant: 196-bp product). The sex of the embryos was determined by detection of the male-specific Sry allele (220 bp product) using the following primers: Sry-Fw6 (5'-TGACTGGGATGCAGTAGTTC-3'), and Sry-Rev6 (5'-TGTGCTAGAGAGAAACCCTG-3'). The PCR amplifications were performed under identical conditions using GoTaq DNA polymerase (Promega). The procedure involved denaturation at $95^{\circ} \mathrm{C}$ for $5 \mathrm{~min}$, followed by 35 cycles of $95^{\circ} \mathrm{C}$ for $30 \mathrm{~s}, 58^{\circ} \mathrm{C}$ for $60 \mathrm{~s}$, and $72^{\circ} \mathrm{C}$ for $30 \mathrm{~s}$, and a final extension at $72^{\circ} \mathrm{C}$ for $7 \mathrm{~min}$. The PCR products were analyzed by electrophoresis in a $1.2 \%$ agarose gel and visualized by ethidium bromide staining. All animal experiments were performed in accordance with institutional guidelines and ethical review committees.

\section{Whole-mount in situ hybridization}

Dissected reproductive organs were fixed with $4 \%$ paraformaldehyde and subsequently with methanol. Rehydrated samples were treated with proteinase $\mathrm{K}(10 \mu \mathrm{g} / \mathrm{ml})$ for $1 \mathrm{~h}$ at room temperature, followed by postfixation with $2 \mathrm{mg} / \mathrm{ml}$ glycine for $30 \mathrm{~s}$ and then $0.2 \%$ glutaraldehyde $/ 4 \%$ paraformaldehyde for $20 \mathrm{~min}$. Subsequently, the samples were processed using an automated InsituPro VS (Intavis AG) according to the manufacturer's protocol. The template for the Lhx1 probe was kindly provided by Dr. R. Behringer. The Wnt9b probe was generated as described (Uchiyama et al., 2010).

\section{Immunohistochemical analysis}

Histological examinations were performed as described previously (Kanda et al., 2014). Mice were fixed in 10\% formalin, embedded in paraffin, and cut into $6-\mu \mathrm{m}$ sections. The Müllerian duct tip was identified by serial horizontal sectioning, and sections within $90 \mu \mathrm{m}$ from the tip were evaluated. Immunostaining was carried out automatically using a BlueMap kit and an automated Discovery System (Roche) or manually for immunofluorescence staining. The following primary antibodies were used: rabbit anti-Pax2 (Covance); mouse anti-Lhx1/2 (Developmental Studies Hybridoma Bank; Cat. No. 4F2); and rabbit anti-Lef1 (Cell Signaling). TdT-mediated dUTP nick end labeling (TUNEL) assays were performed using an ApopTag Plus Fluorescein In situ Apoptosis Detection Kit (Millipore), and the signals were enhanced with Alexa 594-conjugated streptavidin (Invitrogen). For proliferation analyses, embryos were harvested at $1 \mathrm{~h}$ after intraperitoneal BrdU administration ( $0.03 \mathrm{mg} / \mathrm{g}$ body weight). The signals were detected using an anti-BrdU antibody (BD Pharmingen) and an Alexa 594-conjugated secondary antibody. The percentages of BrdU- and TUNEL-positive cells in Müllerian duct epithelia were calculated using at least three pairs of control and mutant embryos (two different sections/embryo). The mean and standard deviation were calculated. Immunofluorescence was visualized with an LSM780 confocal microscope (Carl Zeiss).

\section{Acknowledgments}

We thank Y. Kaku, A. Taguchi, S. Fujimura, and M. Hoshi for helpful advice. This study was supported by KAKENHI (25670413), MEXT, Japan.

\section{References}

BISHOP-CALAME S (1966). Experimental study of the organogenesis of the urogenital system of the chicken embryo. Arc Anat Microsc Morphol Exp 55: 215-309.

CARROLL TJ, PARK JS, HAYASHI S, MAJUMDAR A, MCMAHON AP (2005). Wnt9b plays a central role in the regulation of mesenchymal to epithelial transitions underlying organogenesis of the mammalian urogenital system. Dev Cell9:283-292.
HUANG C-C, ORVIS GD, KWAN KM, BEHRINGER RR (2014). Lhx1 is required in Müllerian duct epithelium for uterine development. Dev Biol 389: 124-136.

IVANOVAA, SIGNORE M, CARO N, GREENE NDE, COPPAJ, MARTINEZ-BARBERA JP (2005). In vivo genetic ablation by Cre-mediated expression of diphtheria toxin fragment A. Genesis 43: 129-135.

KANDA S, TANIGAWA S, OHMORI T, TAGUCHI A, KUDO K, SUZUKI Y, SATO Y, HINO S, SANDER M, PERANTONI AO, SUGANO S, NAKAO M, NISHINAKAMURA R (2014). Sall1 maintains nephron progenitors and nascent nephrons by acting as both an activator and a repressor. J Am Soc Nephrol 25: 2584-2595.

KOBAYASHI A, BEHRINGER RR (2003). Developmental genetics of the female reproductive tract in mammals. Nat Rev Genet 4: 969-980.

KOBAYASHI A, KWAN K-M, CARROLL TJ, MCMAHON AP, MENDELSOHN CL, BEHRINGER RR (2005). Distinct and sequential tissue-specific activities of the LIM-class homeobox gene Lim1 for tubular morphogenesis during kidney development. Development 132: 2809-2823.

LINTON JM, MARTIN GR, REICHARDT LF (2007). The ECM protein nephronectin promotes kidney development via integrin alpha8beta1-mediated stimulation of Gdnf expression. Development 134: 2501-2509.

LITTLE MH, BRENNAN J, GEORGAS K, DAVIES JA, DAVIDSON DR, BALDOCK RA, BEVERDAM A, BERTRAM JF, CAPEL B, CHIU HS, et al., (2007). A highresolution anatomical ontology of the developing murine genitourinary tract. Gene Expr Patterns 7: 680-699.

MADISEN L, ZWINGMAN TA, SUNKIN SM, OH SW, ZARIWALA HA, GU H, NG LL, PALMITER RD, HAWRYLYCZ MJ, JONES AR, LEIN ES, ZENG H (2010). A robust and high-throughput Cre reporting and characterization system for the whole mouse brain. Nat Neurosci 13: 133-140.

MIYAMOTO N, YOSHIDA M, KURATANI S, MATSUO I, AIZAWA S (1997). Defects of urogenital development in mice lacking Emx2. Development 124: 1653-1664.

MURASHIMAA, AKITA H, OKAZAWA M, KISHIGAMI S, NAKAGATAN, NISHINAKAMURA R, YAMADA G (2014). Midline-derived Shh regulates mesonephric tubule formation through the paraxial mesoderm. Dev Biol 386: 216-226.

NISHINAKAMURAR (2008). Stem cells in the embryonic kidney. Kidney Int73:913-917.

OPPELT P, VON HAVE M, PAULSEN M, STRISSEL PL, STRICK R, BRUCKER S WALLWIENER D, BECKMANN MW (2007). Female genital malformations and their associated abnormalities. Fertil Steril 87: 335-342.

ORVIS GD, BEHRINGERRR (2007). Cellular mechanisms of Müllerian duct formation in the mouse. Dev Biol 306: 493-504.

SHAWLOT W, BEHRINGER RR (1995). Requirement for Lim1 in head-organizer function. Nature 374: 425-430.

TORRES M, GÓMEZ-PARDO E, DRESSLER GR, GRUSS P (1995). Pax-2 controls multiple steps of urogenital development. Development 121: 4057-4065.

UCHIYAMAY, SAKAGUCHIM, TERABAYASHIT, INENAGAT, INOUES, KOBAYASH C, OSHIMA N, KIYONARI H, NAKAGATA N, SATO Y, SEKIGUCHI K, MIKI H, ARAKI E, FUJIMURA S, TANAKA SS, NISHINAKAMURA R (2010). Kif26b, a kinesin family gene, regulates adhesion of the embryonic kidney mesenchyme. Proc Natl Acad Sci USA 107: 9240-9245.

WOOLF RB, ALLEN WM (1953). Concomitant malformations; the frequent, simultaneous occurrence of congenital malformations of the reproductive and urinary tracts. Obs Gynecol 2: 236-265.

YOSHINOT, SAITOD, ATSUTAY, UCHIYAMAC, UEDAS, SEKIGUCHIK, TAKAHASH $Y$ (2014). Interepithelial signaling with nephric duct is required for the formation of overlying coelomic epithelial cell sheet. Proc Natl Acad Sci U SA 111:6660-6665.

YU J, CARROLL TJ, MCMAHON AP (2002). Sonic hedgehog regulates proliferation and differentiation of mesenchymal cells in the mouse metanephric kidney. Development 129: 5301-5312. 


\section{Further Related Reading, published previously in the Int. J. Dev. Biol.}

Induction of intermediate mesoderm by retinoic acid receptor signaling from differentiating mouse embryonic stem cells Shiho Oeda, Yohei Hayashi, Techuan Chan, Minoru Takasato, Yuko Aihara,Koji Okabayashi, Kiyoshi Ohnuma and Makoto Asashima Int. J. Dev. Biol. (2013) 57: 383-389 http://dx.doi.org/10.1387/ijdb.130058ma

Revisiting old vaginal topics: conversion of the Müllerian vagina and origin of the "sinus" vagina

Yi Cai

Int. J. Dev. Biol. (2009) 53: 925-934

http://dx.doi.org/10.1387/ijdb.082846yc

The developing female genital tract: from genetics to epigenetics

Julie Massé, Tanguy Watrin, Audrey Laurent, Stéphane Deschamps, Daniel Guerrier and Isabelle Pellerin

Int. J. Dev. Biol. (2009) 53: 411-424

http://dx.doi.org/10.1387/ijdb.082680jm

Charting the course of ovarian development in vertebrates

Kelly A Loffler and Peter Koopman

Int. J. Dev. Biol. (2002) 46: 503-510

http://dx.doi.org/10.1387/ijdb.12141437

Germ cells, gonads and sex reversal in marsupials

$M$ B Renfree and G Shaw

Int. J. Dev. Biol. (2001) 45: 557-567

http://dx.doi.org/10.1387/ijdb.11417899

5 yr ISI Impact Factor $(2013)=2.879$
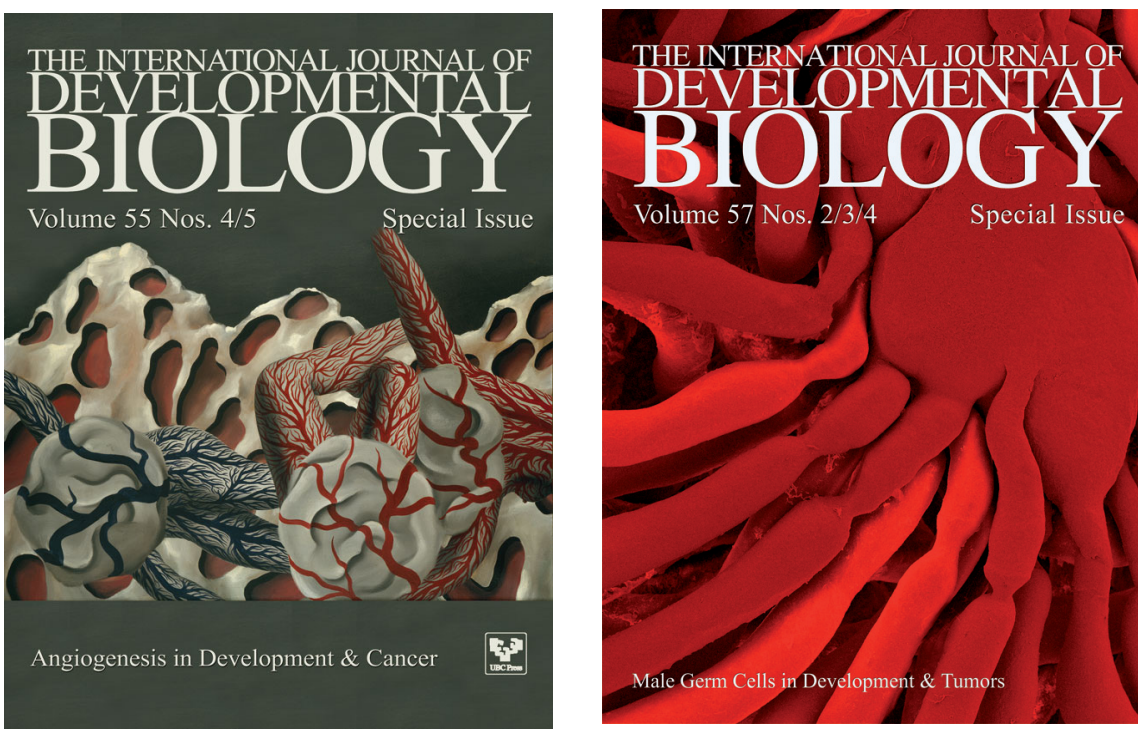

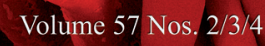

Special Issue

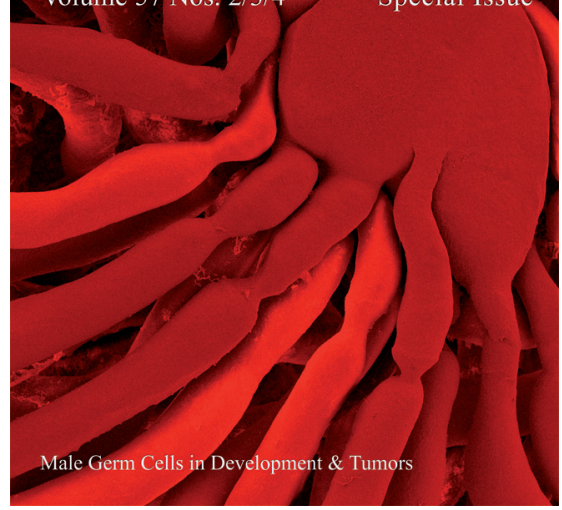

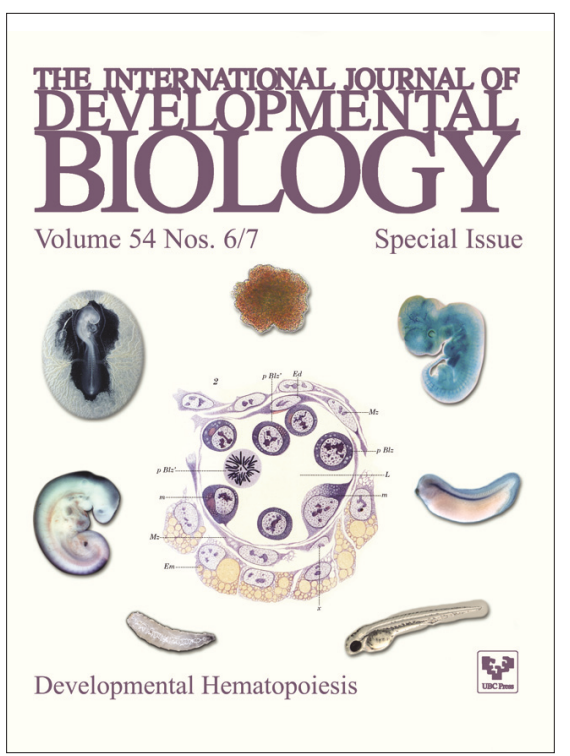

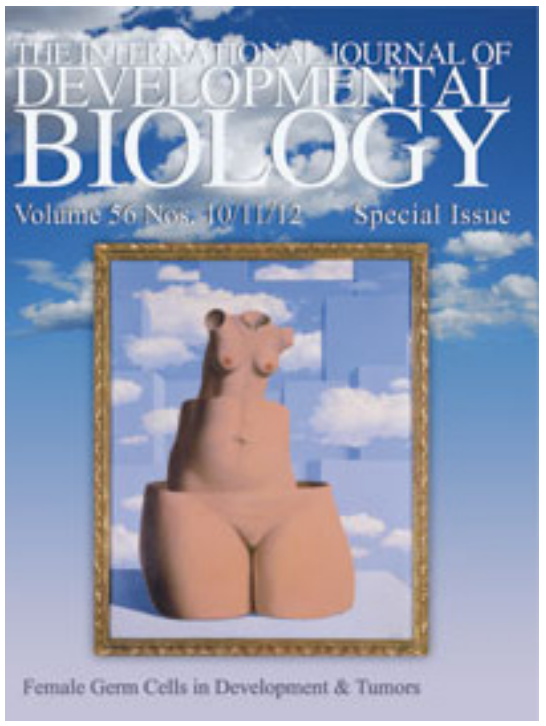

